\title{
Revisiting the Effect of Velocity on Human Force Control
}

\author{
Manikantan Nambi, William R. Provancher, and Jake J. Abbott \\ Department of Mechanical Engineering, University of Utah \\ Salt Lake City UT 84112, USA \\ m.nambi@utah.edu, wil@mech.utah.edu, jake.abbott@utah.edu
}

\begin{abstract}
Human-robot collaborative systems (HRCS) have the potential to dramatically change many aspects of surgery, manufacturing, hazardous-material handling, and other dextrous tasks. We are particularly interested in precise manipulation tasks, which are typically performed under an admittance-control regime, where the controlled velocity is proportional to the user-applied force. During precise fast movements, there is a noticeable degradation in control precision, and prior results have indicated that system velocity, and not system admittance, is the factor that is correlated with force-control precision. In this paper, we report evidence that system admittance is more important than velocity in determining the user's ability to control force, and we provide an explanation as to why prior results might have indicated otherwise.
\end{abstract}

Keywords: Human force control, admittance control.

\section{Introduction}

Human-robot collaborative systems (HRCS) can take the form of teleoperation systems, where movement of a master manipulandum is coupled to movements of a remote slave manipulator, or they can take the form of cooperative systems, where the human directly interacts with a robotic manipulator. For tasks requiring the greatest degree of precision, admittance-type robots are used, which contain a great deal of gearing and inertia such that they appear nonbackdrivable to a human. Such a system is controlled using admittance control: a human interacts directly with a force sensor mounted to the robot, and the robot is computer controlled to move in response to the applied force. The most common and simplest type of admittance control is proportional-velocity control, where the admittance of the system reduces to a simple gain $k$, making the velocity of the robot $V$ linearly proportional to the applied force $F$ :

$$
V=k F
$$

Examples of such systems include the Johns Hopkins University Steady-Hand Robot [1] and the University of Utah Active Handrest [2]. The control law (1) behaves similarly to a "programmable damper" implemented on an impedance-type haptic device (i.e., backdrivable, low inertia), however, it is possible to stably implement much higher levels of damping with an admittance-type device.

A.M.L. Kappers et al. (Eds.): EuroHaptics 2010, Part I, LNCS 6191, pp. 144 1512010.

(c) Springer-Verlag Berlin Heidelberg 2010 
Precise tasks are typically performed at low velocities, but we would like the user to be able to move as fast as possible and still maintain maximum precision. Thus, a better understanding of human force control during admittance control would help us design systems for precise and efficient manipulation. The purpose of this research is to quantify the effects of velocity $(V)$ and admittance gain $(k)$ on human force control precision with visual and haptic feedback.

An 11-15\% mean absolute error was found by Srinivasan and Chen [3] when applying a constant force on a stationary target in the range of $0.25 \mathrm{~N}$ to $1.25 \mathrm{~N}$ with no visual feedback. In the same experiment they found that the error reduced significantly and remained approximately constant at $0.039 \mathrm{~N}$ when visual feedback was provided. Allin et al. [4] found the just noticeable difference (JND) for force with visual feedback to be $10 \%$ for the index finger for a base force of $2.25 \mathrm{~N}$. Lederman et al. [5] studied the force variability with the subject moving their hand at different velocities and under different force levels. They found the end effector, force level, and velocity of the device to have a statistically significant effect on the mean force values.

In the most related prior work, Wu et al. [6] (prior work of one author) studied the effect of velocity and admittance gain on subjects applying force on a moving robotic device. In two separate experiments, the device was controlled under proportional-velocity control and under velocity control (i.e. independent of the applied force), respectively. They determined that the velocity $(V)$, and not admittance gain $(k)$, directly affects force-control precision. However, the conclusion of [6] runs counter to more recent anecdotal experiences in the authors' labs, motivating a reconsideration of the findings. In this paper, we report evidence that, at least for the range of velocities and admittance gains considered here, the conclusion of [6] does not appear to be correct for systems running under proportional-velocity control, and that the admittance gain $(k)$ is in fact the dominant factor in human force control precision. A number of choices made during the design of the experiment in [6] could have contributed to the conclusion. First, $50 \%$ of the data obtained were under velocity control, which may not be representative of performance under proportional-velocity control. Second, in the experiments the controllers were turned on after the human subject reached a desired target force, creating large accelerations at the beginning of each trial that may have affected the results. With real proportional-velocity systems, the velocity naturally ramps up from rest as the human applies force. In addition, the experiments in [6] were conducted with only haptic feedback, requiring the subject to remember a target force value. It is not clear if the discrete event of turning the controller on affected the subjects' ability to remember the target force. In real-life systems, some method of visual feedback conveys information about the amount of applied force. Research by Jones [7] indicates the coefficient of variance for finger and elbow forces to be much lower with both visual and haptic feedback $(4 \%)$ than when only haptic feedback $(12 \%)$ is provided.

\section{Methods}

\subsection{Hardware}

A one degree-of-freedom (1-DOF) robotic device is used to perform the experiment (Fig. 1.a). It consists of a lead-screw-driven linear stage (Servo Systems Co. 


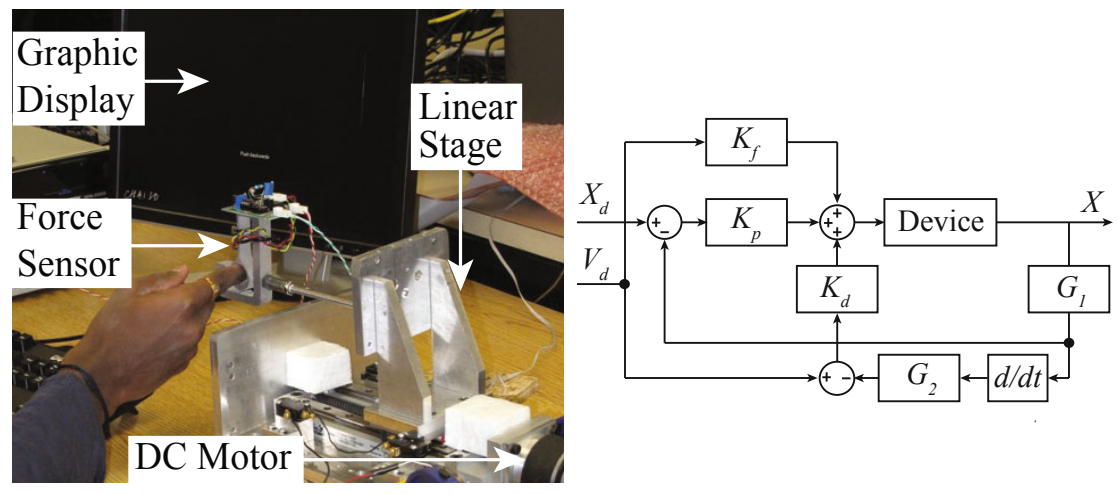

Fig. 1. (a) Experimental apparatus. (b) Control System

MLPS-4-10) with a DC motor (Servo Systems Co. 23SMDC-LCSS). Position feedback is given by an optical encoder mounted on the motor shaft. The lead screw has a pitch of $12.7 \mathrm{~mm}$ and the encoder resolution is 4000 counts/rev after quadrature, which translates to a linear resolution of $3 \mu \mathrm{m}$ for the device. A cantilever-type force sensor was developed for the device. The force sensor has a resolution of $0.7 \mathrm{mN}$ (noise < $0.01 \mathrm{~N}$ ) and is mounted on the linear stage using a rigid rod. A Sensoray 626 DAQ card is used for data acquisition. It has a 16-bit ADC that is used to read force data and a 14bit DAC which is used to command voltages to the current amplifier (Advanced Motion Control 12A8), which is powered by a $24-\mathrm{V}$ linear power supply, and which is used to drive the motor. The software for the device was developed in $\mathrm{C}++$ using the CHAI 3D library. Visual feedback is provided to the subject on the computer screen. The force readings are sampled at $1 \mathrm{kHz}$ and graphics are displayed at $33 \mathrm{~Hz}$.

\subsection{Control Systems}

A PD-plus-feedforward controller has been implemented for the device (Fig. 1. b). UnitDC-gain digital low-pass filters $\mathrm{G}_{1}$ and $\mathrm{G}_{2}$ with time constants $\tau_{1}=0.001 \mathrm{~s}$ and $\tau_{2}=$ 0.0005 are used to reduce quantization error and differentiation noise. The proportional gain $K_{p}$ is set at $30 \mathrm{~V} / \mathrm{mm}$ and derivative gain $K_{d}$ is set at $0.1 \mathrm{~V} \cdot \mathrm{s} / \mathrm{mm}$. The feedforward model for the device was experimentally derived and is given by $K_{f}=0.06 V_{d}+2.2(1-$ $\left.e^{\left(-3.3 V_{d}\right)}\right) \mathrm{V} \cdot \mathrm{s} / \mathrm{mm}$, where $V_{d}$ is the desired velocity. This feedforward model is a smooth function that approximates Coulomb-plus-viscous friction. The inputs for the system are calculated as:

$$
V_{d}(n)=k F(n), \quad X_{d}(n)=X_{d}(n-1)+V_{d}(n) \Delta t
$$

The controlled device is capable of faithfully tracking signals at frequencies below $7 \mathrm{~Hz}$ (44 rad/s), which is sufficient for signals of interest here (Fig. 2). 

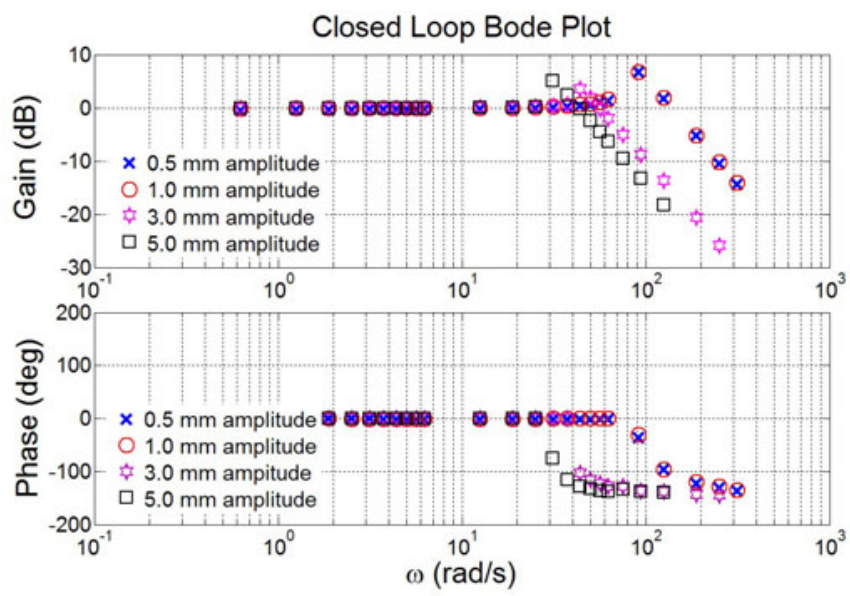

Fig. 2. Closed-loop Bode plot for the system for sinusoidal trajectories in $X_{d}$

\subsection{Pilot Testing}

Using our device and a single subject, we recreated the experiment performed in $\mathrm{Wu}$ et al. [6]. The subject had to apply force on a 1-DOF force sensor (Fig. 1) similar to the one used in [6]. Two experiments, one balanced in force $(F)$ and admittance gain $(k)$ and the other balanced in force $(F)$ and velocity $(V)$ were performed. Our analysis of variance (ANOVA) showed that both $k$ and $\mathrm{F}$ have an effect on the metric (standard deviation of force for the second half of the trial when the device is moving, divided by the standard deviation of force for the first half of the trial when the device is stationary) in the experiment balanced in $k$ and F. For the experiment balanced in $V$ and $F$, only $V$ has an effect on the metric. These results are consistent with those obtained in [6], indicating that it is unlikely that the data collection or analysis in [6] were problematic. Rather, the reasons outlined in Section 1 are the likely causes for their conclusions.

\subsection{Experimental Design}

Our goal is to conduct an experiment that gives information specifically about human force-control precision under the control law (1), rather than about human force control on moving objects in general. An experiment balanced in velocity $(V)$ and admittance gain $(k)$ was designed to test the effect of each factor on human force control precision. Specifically, we are interested in determining when force-control ability is affected by the movement of the device, as compared to force control on a stationary device. The experiment was performed by ten right-handed subjects using their right index fingers. The subjects were all male, with ages ranging from 22 to 37 . Only one of the subjects had prior experience with haptic interfaces. The experiment has institutional-reviewboard approval. Results from pilot studies showed that $V$ and $k$ at which human force control precision is similar to the stationary case occurs for very slow moving systems. Also, limitations in the force sensor restrict the range of values of $V$ and $k$ that can be 
used. Therefore, subjects are tested for values of $V=0.5,0.9,1.6,2.8,5.0 \mathrm{~mm} / \mathrm{s}$ and admittance gains of $k=0.5,0.9,1.6,2.8,5.0 \mathrm{~mm} /(\mathrm{N} \cdot \mathrm{s})$. The target force value to be applied by the subject was calculated using (1). Table 1 shows the target force level for each of the commanded admittance gains to achieve the desired velocity values.

It can bee seen from Table 1 that each combination of $V$ and $k$ has a different target force value associated with it. For each different force value, trials with $k=0$ are also conducted. These values are shuffled to generate a random sequence called a block. Eight such blocks with randomly distributed combinations of $V$ and $k$ are generated for each subject, so we have eight repetitions of each combination. Values of target force and admittance gains in a trial are selected according to these randomly generated blocks for each subject.

During the experiment, subjects are instructed to

Table 1. Force $(\mathrm{N})$ values for different values of $V(\mathrm{~mm} / \mathrm{s})$ and $k(\mathrm{~mm} /(\mathrm{N} \cdot \mathrm{s}))$

\begin{tabular}{|c|c|c|c|c|c|}
\hline$V \backslash k$ & 0.5 & 0.9 & 1.6 & 2.8 & 5.0 \\
\hline 0.5 & 1 & 0.55 & 0.31 & 0.18 & 0.1 \\
\hline 0.9 & 1.8 & 1 & 0.56 & 0.32 & 0.18 \\
\hline 1.6 & .32 & 1.78 & 1 & 0.57 & 0.32 \\
\hline 2.8 & 5.6 & 3.11 & 1.75 & 1 & 0.56 \\
\hline 5.0 & 10 & 5.56 & 3.12 & 1.79 & 1 \\
\hline
\end{tabular}
apply force on the force sensor using their index finger. Their arm is placed perpendicular to the direction of motion of the device with their elbow supported on the table. Subjects had to apply force away from the device (i.e. to their left). Visual feedback is provided on a computer screen in the form of a vertical grey line at the center of the screen indicating the target force to be achieved by the subject. This grey line is surrounded by a green box whose width is set at $\pm 5 \%$ of the target force value. The width of the green box is $15 \mathrm{~mm}$, and the screen is $70 \mathrm{~cm}$ from the subject. The force applied by the subject is displayed by a white vertical line that moves from right to left when force is applied. The force display is scaled such that the center of the screen corresponds to the target force, the right edge corresponds to no force, and the left edge corresponds to twice the target force. Because the target force is always located at the center of the screen, a subject has no indication at the beginning of a trial as to what the target force is, and must use the movement of the white vertical line accompanying their applied force in order to determine the correct force. Subjects are instructed to hit the target force and keep the applied force within the green box to the best of their ability. Each trial lasts for $4 \mathrm{~s}$. The device begins moving at the instant the subject begins to apply force, based on (1). Only the last $2 \mathrm{~s}$ of data are analyzed to remove the effects of the rise time required to achieve the target force (Fig. 3). White noise is played through headphones during the experiment to remove auditory cues. The device is hidden from the subjects by a curtain. Subjects could pause the experiment to rest at any time. Before the experiment, each subject was allowed to interact with the device and experience different force levels and admittance gains.

\section{Results}

We have used two metrics to measure force control precision.

$$
M_{n}=\frac{M}{M_{0}}, \quad S_{n}^{2}=\frac{S^{2}}{S_{0}^{2}}
$$


The first is the normalized mean value of force $M_{n}$, where $M$ is the mean force value for a trial and $M_{0}$ is the mean force value for the static case $(k=0)$ at the same force level in the corresponding block. This metric measures the user's mean force control ability compared to pushing on a stationary sensor. The second metric is the normalized variance in force $S_{n}^{2}$, where $S^{2}$ is the variance for a trial and $S_{0}^{2}$ is the variance for the static case $(k=0)$ at the same force level in the corresponding block. This metric is a measure of the user's tremor, compared to pushing on a stationary sensor. These metrics were specifically chosen to mitigate the effect of force levels, by normalizing all values at a given force level with respect to the static case at that same force level. Using the response of the static case within a given block mitigates bias due to time (fatigue or learning). Because of the design of the metrics, a value of both metrics equal to 1 would mean that the user has a precision similar to that of applying force when the device is stationary, leading to a conclusion that the user's force control is unaffected by the movement of the device.

A fixed-effect ANOVA model is used to test for main effects of $V$ and $k$ on the metrics. The ANOVA shows that both $V$ and $k$ have a main effect on both $S_{n}^{2}$ and $M_{n}$ (p < $0.01)$. The partial effect sizes are given by $\omega_{k}^{2}=0.111$ and $\omega_{V}^{2}=0.009$ for the metric $M_{n}$, and the partial effect sizes for metric $S_{n}^{2}$ are $\omega_{k}^{2}=0.008$ and $\omega_{V}^{2}=0.015$. The results for the metric $M_{n}$ (Fig. 4) show that force control precision degrades rapidly as $k$ increases. We see that the effect of any device motion is that the user's mean force level is reduced (see Fig. 3). It also appears that, at high values of $k$, increasing the velocity has the effect of improving performance (i.e.

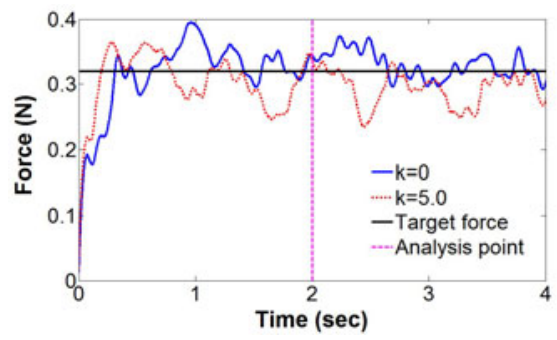

Fig. 3. Typical force-versus-time data for stationary and moving systems. Here, a $0.32-\mathrm{N}$ force is applied by a subject with $\mathrm{k}=0$ and $\mathrm{k}=5.0$. Only the final two seconds of data are used during analysis. bringing $M_{n}$ closer to 1 ). Fig. 5 shows that the metric $S_{n}^{2}$ at high velocities and high admittance gains is noticeably different than at lower velocities and admittance gains. The variance in force is noticeably higher than the static case (i.e. not equal to 1 ) even at the lowest velocities and admittance gains used.

\section{Discussion and Conclusion}

From our statistical analysis it can be seen that both admittance gain $(k)$ and velocity $(V)$ have an effect on force control precision. From Fig. 4, it appears that force control precision degrades rapidly as we increase $k$ for the general population. Wu et al. [6] concluded that only velocity determines human force control precision during admittance control. In this paper we have provided compelling evidence that, at least in the velocity range considered here, both velocity and admittance gain have an effect on force control precision, and that admittance gain has the most pronounced effect. Not all of the tests for force control performed in [6] were conducted using the proportional-velocity control law (1). This could explain the difference in results between the two studies. 


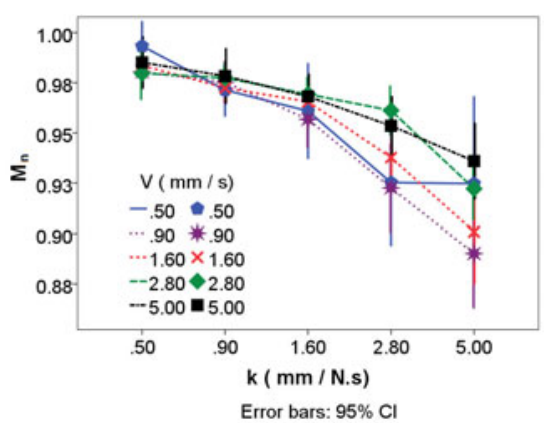

(a)

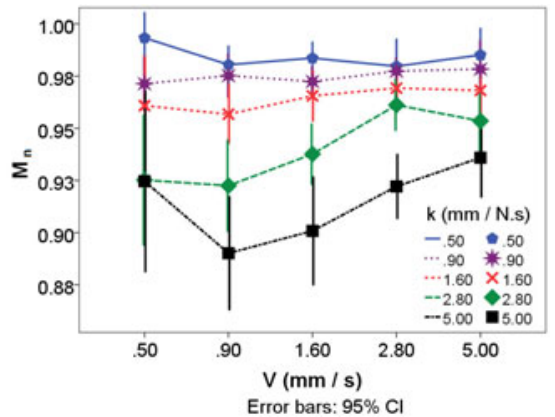

(b)

Fig. 4. Experimental results for metric $M_{n}$ for all 10 subjects combined

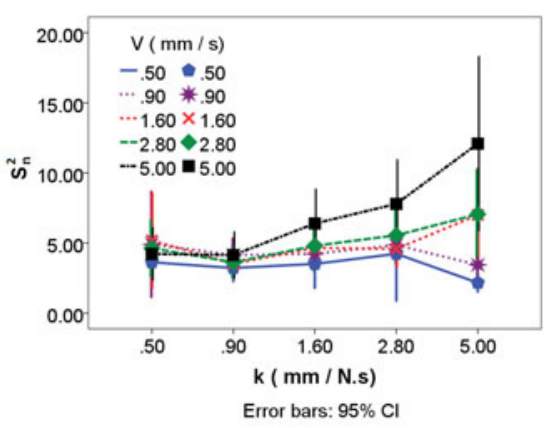

(a)

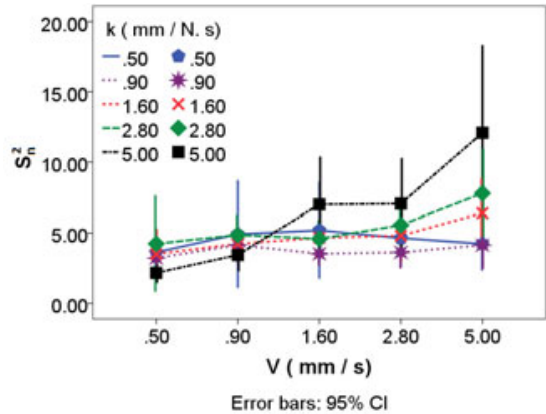

(b)

Fig. 5. Experimental results for metric $S_{n}^{2}$ for all 10 subjects combined

Only for the single data set $\{k=0.5 \mathrm{~mm} / \mathrm{s}, \mathrm{V}=0.5 \mathrm{~mm} / \mathrm{s}\}$ did the data interval for the metrics in our experiment include the value 1 . This implies that even at the lowest velocities and admittance gains used here, the subjects' force control precision is different than the static case. In other words, their force control precision is affected by the movement of the device. Also, from the partial effect sizes we can infer that $11 \%$ of the total variance in metric $M_{n}$ can be explained by $k$, while only $1 \%$ of the total variance is explained by $V$. Thus, the admittance gain $k$ had a predominant effect on human force control precision.

Based on the control law (1), we could also conclude that poor force control at high $k$ and low $V$ is actually a measurement of poor force control at low values of applied force $F$. Although we normalized the metrics by the static case at each force value, in an attempt to control for the effect of changing the applied force level, it may be the case that when the subject is pushing harder and has a stiffer finger, the subject is more robust to movement of the system.

In the future, we will attempt to quantify the effect of admittance gain, velocity, and force level on force control precision. We will attempt to find the conditions 
under which the human user is controlling force with a level of precision equivalent to the isometric (stationary) case. In addition, research indicates that the end effector and gripping configuration has an effect on mean force value while applying force on a stationary target [5]. We intend to study the effect of different gripping configurations on force control precision under different $V$ and $k$. Such a study will help us compare the trends observed in force control presented in this paper with similar results obtained under different common gripping configurations. This would also make the research more applicable to real-life systems where different gripping configurations may be used.

\section{References}

1. Taylor, R., Jensen, P., Whitcomb, L., Barnes, A., Kumar, R., Stoianovici, D., Gupta, P., Wang, Z., de Juan, E., Kavoussi, L.: Steady-hand robotic system for microsurgical augmentation. Int. J. Robotics Research 18(12), 1201-1210 (1999)

2. Fehlberg, M.A., Gleeson, B.T., Leishman, L.C., Provancher, W.R.: Active handrest for precision manipulation and ergonomic support. In: Proc. Symp. Haptic Interfaces for Virtual Environments and Teleoperator Systems, pp. 489-496 (2010)

3. Srinivasan, M.A., Chen, J.S.: Human performance in controlling normal forces of contact with rigid objects. In: Proc. ASME Dynamic Systems and Control Division: Advances in Robotics, Mechatronics, and Haptic Interfaces, pp. 119-125 (1993)

4. Allin, S., Matsuoka, Y., Klatzky, R.: Measuring just noticeable differences for haptic force feedback: Implications for rehabilitation. In: Proc. Symp. Haptic Interfaces for Virtual Environments and Teleoperator Systems, pp. 299-302 (2002)

5. Lederman, S.J., Howe, R.D., Klatzky, R.L., Hamilton, C.: Force variablitiy during surface contact with bare finger or rigid probe. In: Proc. Symp. Haptic Interfaces for Virtual Environments and Teleoperator Systems, pp. 154-160 (2004)

6. Wu, M., Abbott, J.J., Okamura, A.M.: Effect of velocity on human force control. In: Proc. Joint Eurohaptics Conf. and Symp. Haptic Interfaces for Virtual Environment and Teleoperator Systems, pp. 73-79 (2005)

7. Jones, L.A.: Visual and haptic feedback in the control of force. Experimental Brain Research 130, 269-272 (2000) 